\title{
DEVELOPPEMENT DU SYSTEME RACINAIRE CHEZ LE PALMIER A HUILE SELON L'ORIGINE GENETIQUE ET LE REGIME HYDROPOTASSIQUE DU SOL
}

\author{
L. NODICHAO'1, S. AKE ${ }^{2}$ et C. JOURDAN ${ }^{3}$ \\ ${ }^{1}$ Centre de Recherches Agricoles sur les Plantes Pérennes (CRA-PP), BP. 01 Pobè, Bénin \\ ${ }^{2}$ Université de Cocody-Abidjan, Laboratoire de Physiologie et Pathologie Végétales,UFR Biosciences, \\ 22 B.P. 582 Abidjan 22, Côte d'Ivoire \\ ${ }^{3}$ CIRAD-CP, TA 80/01, 34398 Montpellier cedex 5
}

\begin{abstract}
RESUME
L'architecture du système racinaire du palmier à huile joue un rôle important dans la résistance à la sécheresse, grâce à la mobilisation de l'eau et des sels minéraux. Le système racinaire des plants issus de 3 croisements de palmier à huile $\mathrm{B}, \mathrm{C}$ et $\mathrm{E}$ a été étudiée. Les plants ont été transférés dans des tubes PVC (95 cm de profondeur $\times 10 \mathrm{~cm}$ de diamètre). Chaque tube PVC a consisté en un assemblage de 6 petits tubes. Deux régimes hydriques et deux niveaux de potassium (K) ont été testés. Les effets des croisements et de l'interaction "croisement $\mathrm{x}$ niveau de $\mathrm{K}$ x régime hydrique" ont été significatifs pour les paramètres telles la longueur totale, la surface totale et la matière sèche des racines. Ces résultats montrent que pour plus de progrès dans la sélection du système racinaire adapté à la sécheresse, les sélectionneurs gagneraient à prendre en compte à la fois la sécheresse la déficience potassique.
\end{abstract}

Mots clés : Palmier à huile, déficit hydrique, déficience potassique, architecture racinaire, plasticité racinaire.

\section{ABSTRACT}

DEVELOPMENT OF ROOT SYSTEM OF OIL PALM DEPENDING ON GENETIC ORIGIN AND SOIL HYDROPOTASSIC REGIME

Root system architecture is important in drought resistance, because of its role in water and nutrient mobilizations. The variability of root system architecture of 3 oil palm crosses $B, C$ and $E$ was studied. The 3 crosses were different for their sensitivity to drought. The plants were potted into plastic PVC tubes $(95 \mathrm{~cm}$ in depth $\times 10 \mathrm{~cm}$ in diameter). Each PVC tube was constructed with 6 small tubes assembled together. Two soil moistures regimes and two levels of potassium $(K)$ were tested. For root length, surface area and dry matter, effects of crosses and "cross $x$ K levels $x$ water regime" interactions were significant. The results suggest that breeders could make more progress in selection of root system adapted to drought, if they consider simultaneously both drought and $K$ deficiency parameters.

Key words : Oil palm, drought, potassium deficiency, root architecture, root plasticity.

\section{INTRODUCTION}

L'énergie solaire utilisée dans les processus de photosynthèse et de transpiration est absorbée par les organes aériens, tandis que l'absorption de l'eau et des sels minéraux est exclusivement assurée par les racines (Kolek et Kozinka,
1992). En outre, les seuls organes qui réagissent directement à l'état hydrique du sol et le communiquent aux organes aériens, sont les racines (Davies et Bacon, 2003). Elles constituent les principales portes d'entrée des sels minéraux et de l'eau nécessaires à la croissance et à la production des plantes. Il existe au niveau des membranes racinaires des canaux passifs de 
transport d'eau et des sels minéraux, «aquaporins» dont les activités régulent la conductance hydraulique des racines (Vandeleur et al., 2005). Cependant, la conductance hydraulique racinaire et l'absorption des éléments minéraux ne dépendent pas que des caractéristiques anatomiques des racines (Kolek et Kozinka, 1992). Les caractéristiques morphologiques des systèmes racinaires déterminent également la capacité d'absorption de l'eau et des éléments minéraux. La densité racinaire conditionne l'absorption de l'eau et des minéraux (Chopart et Siband, 1999 ; Tinker et Nye, 2000 ; Huang et Gao, 2000). Ainsi, Pardo et al. (2000) trouvent des liens entre la structure du sol, la distribution des racines et l'absorption de l'eau chez Cicer arietinum. L'intérêt des caractères morphologiques ou anatomiques des racines du palmier à huile, pour l'absorption de l'eau ou des minéraux a été étudié par plusieurs auteurs (Tailliez, 1971 ; Reis de Carvalho, 1991 ; Jourdan, 1995). Ruer (1968) a étudié la morphologie et l'anatomie des racines du palmier à huile, en particulier la distribution des zones absorbantes. Les résultats obtenus ont, par ailleurs, mis en évidence une influence du patrimoine génétique et des facteurs externes comme l'humidité et la fertilité du sol sur la croissance des racines. Des travaux plus récents de caractérisation morphologique et anatomique du système racinaire ont permis de décrire de façon détaillée l'architecture du système racinaire du palmier à huile et de la modéliser (Jourdan et al., 1995 ; Jourdan et Rey, 1997a, 1997b). Cornaire et al. (1994) ont rapporté que les croisements du palmier à huile tolérants à la sécheresse ont un système racinaire plus développé que celui des croisements sensibles. Les palmiers à système racinaire développé pourraient avoir un avantage morphologique pour la mobilisation des minéraux comme le potassium qui est un élément essentiel pour accroître le rendement chez le palmier à huile (Quencez et De Taffin, 1981). Cet avantage morphologique peut être précieux pendant la sécheresse où l'absorption des minéraux devient difficile à cause de la réduction de leur mobilité (Tinker et Nye, 2000).

Cependant, les travaux antérieurs sur le système racinaire du palmier à huile, ont concerné la matière sèche (Anonyme, 1992 ; Cornaire et al., 1994 ; Khalid et al., 1999) plutôt que des caractères directement liés aux phénomènes d'absorption hydrique ou minérale (surface, longueur, ou diamètre). La possibilité de faire des analyses d'images (Costa et al., 2000 ; Danjon et al., 2000) permet de mesurer aujourd'hui avec précision la surface totale, la longueur totale ou le diamètre des racines des végétaux.

L'objectif de cette étude est de caractériser la variabilité génétique du système racinaire de trois croisements de palmier à huile sous différents traitements hydropotassiques.

\section{MATERIEL ET METHODES}

\section{MATERIEL VEGETAL}

Trois croisements de palmier à huile ont été utilisés pour l'étude. Les croisements sont issus de 3 catégories C1001F, C6446 et C2001, de semences obtenues auprès du Centre de Recherches Agricoles sur les Plantes Pérennes de Pobè (Bénin). Leur résistance présumée à la sécheresse et l'origine parentale sont présentées dans le tableau 1.

Tableau 1 : Résistance présumée de 3 croisements de palmier à huile à la sécheresse selon l'origine des parents et la catégorie des semences.

Expected drought resistance of 3 oil palm crosses according to their genetic origin and seed category.

\begin{tabular}{|c|c|c|c|c|c|}
\hline \multirow{2}{*}{ Catégorie } & Origine & \multicolumn{2}{|c|}{ croisements } & \multirow{2}{*}{$\begin{array}{l}\text { Résistance } \\
\text { présumée }\end{array}$} & \multirow{2}{*}{ Code } \\
\hline & Dura x Ténéra & femelle & Mâle & & \\
\hline $\mathrm{C} 1001 \mathrm{~F}$ & DA115D x LM2T & PO3179D & PO2558P & Intermédiaire & B \\
\hline C6446 & DA8D x (LM9T x LM13T) & PO3724D & PO3643P & résistant & $\mathrm{C}$ \\
\hline C2001 & $(\mathrm{LM} 404 \mathrm{D} \times \mathrm{DA} 10 \mathrm{D}) \times \mathrm{LM} 2 \mathrm{~T}$ & PO4672D & PO2558P & Sensible & E \\
\hline
\end{tabular}




\section{Matériel d'expérimentation}

L'appareil d'expérimentation est un assemblage de petits tubes en $P V C$ de $10 \mathrm{~cm}$ de diamètre. Chaque assemblage comporte 5 tubes de PVC de $15 \mathrm{~cm}$ et un tube de $20 \mathrm{~cm}$, de profondeur. Le tube de $20 \mathrm{~cm}$ a été placé à la base des 5 tubes de $15 \mathrm{~cm}$. Les 6 tubes ont été assemblés par des demis cylindres de PVC de $15 \mathrm{~cm}$ de long et attachés au fil de fer. Chaque appareil forme ainsi un tube de $95 \mathrm{~cm}$ de profondeur, fermé à la base par une toile nylon de $1 \mathrm{~mm}$ de maille doublée. Une couche de $2 \mathrm{~cm}$ de gravillons a été déposée au fond du tube pour faciliter le drainage. Le dispositif a permis de disposer plus facilement du sol contenu dans chaque petit tube en vue de collecter toutes les racines qui s'y trouvent.

\section{METHODES}

\section{Installation de la prépépinière}

La germination des graines a été effectuée suivant la méthode classique de l'Institut de Recherches pour les Huiles et Oléagineux (IRHO) (Corrado et Wuidart, 1990). Des graines germées de 1,5 à $2 \mathrm{~cm}$ de longueur radiculaire ont été triées et semées dans des petits pots de polyéthylène de $20 \mathrm{~cm}$ de profondeur $x 8,5$ $\mathrm{cm}$ de diamètre. Chaque pot a été rempli de terreau jusqu'à $3 \mathrm{~cm}$ de son bord supérieur. Les plantules ont été placées sous ombrage pendant toute la phase de prépépinière et ont été arrosées chaque matin entre $7 \mathrm{~h} 30$ et $8 \mathrm{~h}$. La culture en prépépinière a duré $50-60 \mathrm{j}$. Après la prépépinière les plantules ont été transférées en serre dans des tubes en chlorure de polyvinyle (PVC).

\section{Transfert dans les tubes PVC}

Au moment du transfert, la hauteur, le diamètre du pseudobulbe et le nombre de feuilles des plantules ont été mesurés, ce qui a permis de retenir les plants de taille similaire. Les plantules ont été ensuite transférées dans les tubes PVC. Chaque tube a reçu $9 \mathrm{~kg}$ de sol tassé à une densité de 1,2 à $1,3 \mathrm{~g} \mathrm{~cm}^{-3}$. Après le transfert de la plantule, le sol a été immédiatement saturé d'eau et soumis au drainage pendant 3 à $4 \mathrm{j}$. La surface du sol a été protégée par une toile plastique de polyéthylène noire pour minimiser les pertes d'eau par évaporation du sol. Chaque plantule a été arrosée par jour avec une quantité d'eau équivalente à l'évapotranspiration constatée dans son tube en $24 \mathrm{~h}$. Ce régime hydrique a été appliqué jusqu'à l'application d'un régime de restriction hydrique. Les températures sous serre où les plantules ont été transférées, sont supérieures de 4 à $6{ }^{\circ} \mathrm{C}$ aux températures ambiantes dans la journée. Les températures maxima et minima de l'air ambiant durant l'essai ont varié de 25,8 à $38,2^{\circ} \mathrm{C}$, et de 20,2 à $26,8^{\circ} \mathrm{C}$, respectivement.

\section{Dispositif expérimental et traitements}

L'étude a porté sur les 3 facteurs suivants :

- Origine génétique (DA115D x LM2T, DA8D $x$ (LM9T x LM13T) et (LM404 x DA10D) x LM2T)

- Régime potassique (apport de $\mathrm{KCl}(\mathrm{K})$ et sans apport de $\mathrm{KCl}(\mathrm{KO})$ ) ;

- Régime hydrique (sans déficit hydrique $(\mathrm{N})$ et avec déficit hydrique (S)).

Les 3 facteurs ont été combinés pour déterminer 12 traitements (Tableau 3). Les douze traitements ont été attribués à 48 tubes en PVC assemblés comme décrit. Les 48 tubes ont été disposés sur des chassies en bois suivant un dispositif en bloc de Fisher avec quatre répétitions.

Chaque origine génétique a été représentée par un croisement (Tableau 1). Les deux niveaux de régime potassique ont été réalisés à partir de deux solutions minérales (Tableau 2). Celles-ci ont été ajoutées à du sol prélevé dans une ancienne palmeraie. La teneur initiale du sol prélevé en K est de 0,16 méq/100 g. Quatre jours après le transfert, chaque plant a bénéficié d'un arrosage quotidien équivalent à l'évapotranspiration (ETR) dans son tube jusqu'au $104^{\mathrm{e}} \mathrm{j}$ après le transfert. L'ETR a été déterminée suivant la technique des pesées successives (Sinclair et Ludlow, 1986 ; Egilla et al., 2001). A partir, du $105^{\mathrm{e}} \mathrm{j}$ après le transfert des plants, deux régimes hydriques $N$ et $S$ ont été appliqués. A cet effet, chaque plant sous régime de déficit hydrique, a été quotidiennement soumis à un stress hydrique $S$ équivalent à la plus faible valeur d'ETR observée durant les $24 \mathrm{~h}$ avant l'arrosage du jour. Le régime de restriction hydrique a été appliqué jusqu'à faire baisser la réserve d'eau du sol (RU) à un niveau résiduel de $50 \%$ dans les tubes sous régime $\mathrm{S}$. Ce niveau de déficit hydrique a été atteint au bout de $29 \mathrm{j}$ d'application du régime $S$. Chaque plant sous régime $\mathrm{N}$ a été arrosé par jour avec une quantité d'eau égale à l'évapotranspiration 
en $24 \mathrm{~h}$ dans son tube pour maintenir l'humidité du sol à un niveau équivalent à $100 \%$ RU. La balance utilisée pour quantifier la perte d'eau des tubes a une portée de $35 \pm 0,002 \mathrm{~kg}$.

Tableau 2 : Quantité d'éléments $\mathrm{K}$ ajoutée au sol selon le traitement.

Quantity of K added to soil according to treatments.

\begin{tabular}{|c|c|c|}
\hline \multirow{3}{*}{ Sel minéral } & Traitement sans K & Traitement avec $\mathrm{K}$ \\
\hline & (K0) & $(\mathrm{K})$ \\
\hline & \multicolumn{2}{|c|}{$\mathrm{g} /$ tube } \\
\hline Urée & 0,008 & 0,008 \\
\hline Diammonium hydrogénophosphate & 0,003 & 0,003 \\
\hline Chlorure de potassium & 0 & 0,002 \\
\hline
\end{tabular}

Tableau 3 : Traitements d'origine génétique et le régime hydropotassique appliqués dans un essai factoriel de 3 croisements du palmier sous deux régimes potassiques et deux régimes hydriques.

Treatments applied in a factoriel design with 3 crosses of oil palm, two hydropotassic regimes and two water regimes.

\begin{tabular}{lcccc}
\hline \multirow{2}{*}{$\begin{array}{l}\text { Origine } \\
\text { génétique } \\
\text { (croisements) }\end{array}$} & \multicolumn{2}{c}{ Régime hydrique sans déficit hydrique $(\mathrm{N})$} & \multicolumn{2}{c}{ Régime hydrique avec déficit hydrique (S) } \\
\cline { 2 - 5 } & $\begin{array}{c}\text { Avec apport de } \mathrm{KCl} \\
(\mathrm{K})\end{array}$ & $\begin{array}{c}\text { Sans apport de } \mathrm{KCl} \\
(\mathrm{K} 0)\end{array}$ & $\begin{array}{c}\text { Avec apport de } \mathrm{KCl} \\
(\mathrm{K})\end{array}$ & $\begin{array}{c}\text { Sans } \\
\text { apport de } \mathrm{KCl} \\
(\mathrm{K} 0)\end{array}$ \\
\hline $\mathrm{B}$ & $\mathrm{BNK}$ & $\mathrm{BNK} 0$ & $\mathrm{BSK}$ & BSK0 \\
$\mathrm{C}$ & $\mathrm{CNK}$ & $\mathrm{CNK} 0$ & $\mathrm{CSK}$ & CSK0 \\
$\mathrm{E}$ & ENK & ENK0 & ESK & ESK0 \\
\hline
\end{tabular}

B : DA115D x LM2T, C : DA8D x (LM9T x LM13T), E : (LM404 x DA10D) x LM2T

\section{Observations et mesures}

Outre le suivi quotidien de l'évapotranspiration, la hauteur des plants et le nombre de feuilles par plant, ont été mesurés toutes les semaines. A la fin de 29 j de restriction hydrique, les racines contenues dans chaque horizon ont été collectées et lavées dans de petits bacs, puis rincées avec de l'eau distillée. La longueur de la racine primaire $(\mathrm{RI})$ la plus longue ainsi que le nombre de racines $\mathrm{RI}$ ont été notés. La surface et la longueur totale des racines ainsi que le nombre de ramifications, ont été déterminés avec le logiciel WINRHIZO de Régent Instruments Inc. Version $4.1 \mathrm{c}$, à partir d'images racinaires scannées avec un scanner EPSON LA1600. Les analyses statistiques ont été effectuées avec le logiciel Genstat version 4.2. Les paramètres décrivant le développement global du système racinaire (longueur totale, la surface totale, les nombres de ramifications et de RI, la matière sèche totale, la longueur totale selon les différentes classes de racines) ont été soumis à une analyse de variance en utilisant la directive de structure de blocs randomisés de Genstat. L'analyse de variance de la surface racinaire par horizon (surface totale des racines par tranche de sol) a été réalisée en faisant une partition des sources de variations en fonction de la profondeur d'enracinement. Le test de StudentNewman-Keuls a été utilisé pour comparer les moyennes des paramètres décrivant le développement global du système racinaire. Une courbe d'évolution de la surface racinaire en fonction de la profondeur a été utilisée pour illustrer les interactions «profondeur $x$ génotype $x$ dose de $\mathrm{KCl}$ ».

\section{Analyse d'images racinaires}

La technique consiste à obtenir à partir d'une source (scanner, vidéo caméra, fichier d'images préenregistrées) des images d'objets sur l'écran d'un ordinateur, puis à analyser les images projetées à l'écran avec le logiciel WINRHIZO. Le logiciel permet de déterminer plusieurs caractères racinaires dont la longueur totale, la surface totale, le diamètre moyen des racines 
et le nombre total de ramifications racinaires. Le logiciel permet aussi de déterminer la longueur totale et la surface totale des racines de différentes classes en fonction de leur diamètre ou leur couleur (Régent Instruments Inc., 2001). Dans le cadre de ce travail, les différentes classes racinaires ont été fixées en fonction de leur diamètre. Les racines collectées ont été soigneusement débarrassées de la terre sous faible pression d'eau au dessus de deux tamis à maille très fine $(<2 \mathrm{~mm})$ pour éviter des pertes de racines fines. Les racines ont été lavées de nouveau avec de l'eau distillée pour enlever toute particule de sol et autres débris avant l'analyse. Elles ont été ensuite transportées au laboratoire où elles ont été conservées au congélateur sous une température de $5^{\circ} \mathrm{C}$ pour une durée maximale de 3 semaines. Avant l'analyse des images, les racines ont été trempées dans une solution de bleu de toluidine de même concentration $(0,1 \mathrm{~g} / 100 \mathrm{ml})$ que celle utilisée par Costa et al. (2000) pendant 10 à $15 \mathrm{mn}$. Ceci pour accroître le contraste de l'image des racines à l'écran et améliorer la précision de l'analyse. Les échantillons de racines ont été analysés par tranche de sol. Ainsi, toutes les racines collectées dans une même couche de sol ont été étalées dans un plateau en plexiglas de $40 \times 30 \times 2 \mathrm{~cm}$ fourni par le concepteur de WINRHIZO. Lorsque la longueur totale des racines collectées dépasse $5 \mathrm{~m}$, l'échantillon racinaire est subdivisé pour assurer un bon étalement des racines dans le plateau. Ceci permet de réduire le temps nécessaire à l'analyse de l'image et les erreurs liées aux superpositions de plusieurs racines. Chez le palmier à huile il n'a pas été nécessaire de mettre de l'eau dans le plateau en plexiglas comme chez les céréales pour démêler les racines (Nodichao, 1997 ; Costa et al., 2000). Le plateau contenant les racines ainsi préparées a été déposé sur un scanner EPSON LA 1600 qui procède à la numérisation et à la projection de l'image sur l'écran d'un ordinateur auquel il est branché. L'image projetée à l'ordinateur a été ensuite analysée avec le logiciel WINRHIZO. Les données retenues pour les analyses statistiques sont celles calculées suivant la méthode du concepteur de WINRHIZO.

\section{RESULTATS}

\section{PARAMETRES DE CROISSANCE GLOBALE DU SYSTEME RACINAIRE}

La surface totale (SRT), la longueur totale (LRT), le nombre total de ramifications et la matière sèche (MSR) des racines, ont été influencés de façon significative $(p<5 \%)$ par les interactions croisement $x$ niveau $K$ (sol) ou croisement $x$ régime hydrique (eau) ou croisement $x$ sol $x$ eau (Tableau 4). Le nombre moyen de RI a été respectivement de $6,49,6,75$ et 6,38 pour les croisements $B, C$ et $E$. L'analyse de variance n'a pas révélé de différence significative pour ce caractère.

\section{Longueur totale et surface totale de racines}

La LRT et la SRT du croisement E ont été inférieures à celles des croisements $C$ et $B$ dans les tubes sans déficit hydrique (Tableau 5). Cependant, l'amplitude de la différence entre les trois croisements varie suivant le régime potassique. Les différences entre le croisement $E$ et les croisements $B$ et $C$ ont été significatives sous déficit potassique. Les longueurs totales de racines des croisements $B$ et $C$ sous le régime hydropotassique sans déficit hydrique et sans apport potassique ont dépassé celle du génotype $E$ de $7,9 \mathrm{~m}$ et 7,3 $\mathrm{m}$, tandis que dans les tubes sous régime hydropotassique NK (sans déficit hydrique avec apport de $\mathrm{KCl}$ ) la différence de longueur racinaire entre les croisements $B$ ou $C$ et le croisement $E$ a été de $4,6 \mathrm{~m}$ et 2,7 $\mathrm{m}$ (Tableau 5). Sous déficit hydrique, les relations entre la LRT ou SRT du croisement $E$ et celles des croisements $B$ et $C$ ont varié aussi selon la dose de K (Tableau 5). Les différences entre la LRT ou la SRT des croisements $B$ et $C$ ont été significatives sous le double déficit hydropotassique (avec déficit hydrique et sans apport de $\mathrm{KCl}$ ), alors que les LRT et SRT du croisement $E$ ont été intermédiaires entre celles de $B$ et $C$ (Tableau 5). Pour ce régime hydropotassique la longueur totale des racines du croisement $B$ a été inférieure à celle des croissements $C$ et $E$ de $8 \mathrm{~m}$ et $4,7 \mathrm{~m}$, et la différence de surface totale des racines, a été de $179 \mathrm{~cm}^{2}(C)$ et $89 \mathrm{~cm}^{2}(E)$. 
Tableau 4 : Analyse de variance (ANOVA) (Carrés Moyens) de différents paramètres descriptifs de l'architecture racinaire de trois croisements de palmier à huile selon les traitements potassiques et les régimes hydriques.

ANOVA (Means Squares) of different parameters of root architecture of three oil palm crosses according to $\mathrm{K}$ levels and water regimes.

\begin{tabular}{lcccccccccc}
\hline $\begin{array}{l}\text { Source } \\
\text { variation }\end{array}$ & d.l & $\begin{array}{c}\text { Longueur } \\
\text { totale }\end{array}$ & $\begin{array}{c}\text { Surface } \\
\text { totale }\end{array}$ & $\begin{array}{c}\text { Nombre } \\
\text { ramif.*100 }\end{array}$ & $\begin{array}{c}\text { Longueur } \\
0<\phi \leq 0,5\end{array}$ & $\begin{array}{c}\text { Longueur } \\
0,5<\phi \leq 1\end{array}$ & $\begin{array}{c}\text { Longueur } \\
1<\phi \leq 3\end{array}$ & $\begin{array}{c}\text { Longueur } \\
\phi>3\end{array}$ & $\begin{array}{c}\text { Matière } \\
\text { sèche } \\
\text { racine }\end{array}$ & $\begin{array}{c}\text { Nombre } \\
\text { RI }\end{array}$ \\
\hline Blocs & 3 & 632673 & 46602 & 1975,7 & 158030 & 62555 & 52134 & 246,1 & 0,551 & 3,955 \\
Croissement & 2 & $471666^{*}$ & $38920^{*}$ & 251,6 & 93911 & $118809^{* *}$ & $30440^{*}$ & 239,0 & $1,461^{* *}$ & 0,567 \\
Sol & 1 & 171811 & 2967 & 2,6 & 70222 & 6179 & 560 & 128,8 & 0,006 & 0,085 \\
Eau & 1 & 9390 & 150 & 55,9 & 2099 & 4473 & 1041 & 0,3 & 0,046 & 0,134 \\
Géno x sol & 2 & $544118^{*}$ & 15106 & 1114,6 & $236573^{*}$ & 27134 & 12567 & 87,9 & 0,015 & 0,563 \\
Géno x eau & 2 & 437182 & 18456 & $2471,5 * *$ & 133140 & $65062^{*}$ & 16032 & 84,7 & $1,162^{* *}$ & 0,084 \\
Sol x eau & 1 & 39955 & 7291 & 21,4 & 1869 & 6764 & 7274 & 3,2 & 0,006 & 0,891 \\
Géno x sol & 2 & $494462^{*}$ & $43886^{* *}$ & 669,7 & 108734 & $70505^{*}$ & $34838^{*}$ & $452,2 *$ & $0,547^{*}$ & 3,088 \\
x eau & & & & & & & & & & \\
Erreur & 33 & 136454 & 7580 & 381,4 & 45792 & 19430 & 7201 & 116,8 & 0,146 & 1,595 \\
\hline
\end{tabular}

** : significative au seuil 0,01 ; * significative au seuil $5 \%$; les valeurs sans astérisque ne sont pas significatives au seuil $5 \%$; d.I. : degré de liberté $; \phi$ : diamètre racinaire

Tableau 5 : Longueur, surface et matière sèche de racines par plant chez le palmier à huile selon l'origine génétique et le régime hydropotassique.

Roots length, surface area and dry matter per oil palm plant according to genetic origin and hydropotassic treatments.

\begin{tabular}{|c|c|c|c|c|c|c|}
\hline \multirow{2}{*}{$\begin{array}{l}\text { Caractères } \\
\text { racinaires }\end{array}$} & \multirow{2}{*}{$\begin{array}{l}\text { Origine } \\
\text { génétique }\end{array}$} & \multicolumn{2}{|c|}{$\begin{array}{c}\text { Régime hydrique sans déficit } \\
\text { hydrique }(\mathrm{N})\end{array}$} & \multicolumn{2}{|c|}{$\begin{array}{l}\text { Régime hydrique avec déficit } \\
\text { hydrique (S) }\end{array}$} & \multirow[t]{2}{*}{ moyenne } \\
\hline & & Avec $\mathrm{KCl}(\mathrm{K})$ & Sans $\mathrm{KCl}(\mathrm{K} 0)$ & Avec $\mathrm{KCl}(\mathrm{K})$ & Sans $\mathrm{KCl}(\mathrm{K} 0)$ & \\
\hline Longueur totale & B & $19,32 \mathrm{a}$ & $21,81 \mathrm{a}$ & $20,62 \mathrm{a}$ & $13,84 b$ & 18,90 \\
\hline \multirow{2}{*}{$\begin{array}{l}\text { Root length } \\
\text { (m/plant) }\end{array}$} & $\mathrm{C}$ & $17,43 a$ & $21,14 a$ & $15,26 \mathrm{a}$ & $21,86 a$ & 18,92 \\
\hline & $\mathrm{E}$ & $14,77 \mathrm{a}$ & $13,88 b$ & $16,53 \mathrm{a}$ & $18,57 \mathrm{ab}$ & 15,94 \\
\hline Surface totale & B & $382 \mathrm{a}$ & $488 \mathrm{a}$ & $459 \mathrm{a}$ & $274 b$ & 401 \\
\hline \multirow{2}{*}{$\begin{array}{l}\text { Root surface } \\
\text { area }\left(\mathrm{cm}^{2} / \text { plant }\right)\end{array}$} & $\mathrm{C}$ & $380 \mathrm{a}$ & $420 \mathrm{a}$ & $328 \mathrm{a}$ & $453 a$ & 395 \\
\hline & E & $291 \mathrm{a}$ & $267 \mathrm{~b}$ & $330 \mathrm{a}$ & $363 \mathrm{ab}$ & 313 \\
\hline \multirow{2}{*}{$\begin{array}{l}\text { Matière sèche } \\
\text { totale }\end{array}$} & B & $1,55 \mathrm{~b}$ & $1,96 \mathrm{a}$ & $1,96 a$ & $1,56 \mathrm{a}$ & 1,76 \\
\hline & $\mathrm{C}$ & $2,64 \mathrm{a}$ & $2,35 \mathrm{a}$ & $1,74 \mathrm{a}$ & $1,98 \mathrm{a}$ & 2,18 \\
\hline $\begin{array}{l}\text { Dry matter } \\
\text { (g/plant) }\end{array}$ & E & $1,43 b$ & $1,31 b$ & $1,66 \mathrm{a}$ & $1,95 \mathrm{a}$ & 1,59 \\
\hline
\end{tabular}

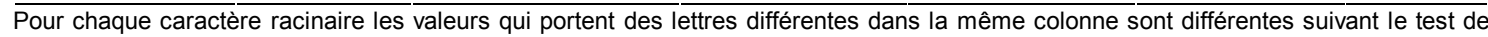
Student-Newman-Keuls à $5 \%$. B : DA115D x LM2T, C : DA8D x (LM9T x LM13T), E : (LM404 x DA10D) x LM2T 


\section{Production de matière sèche racinaire}

Les interactions "génotype $x$ sol $x$ eau» ont été significatives pour la MSR comme pour la LRT et la SRT (Tableau 4). Cependant, contrairement à la LRT ou la SRT, la différence de matière sèche totale de racines entre le croisement $C$ et les croisements $B$ et $E$ a été significative sous le régime hydropotassique NK (Tableau 5). Le croisement $\mathrm{C}$ a produit par plant $1,09 \mathrm{~g}$ et $1,21 \mathrm{~g}$ de matière sèche racinaire de plus que les croisements $B$ et $E$. La différence entre le croisement $\mathrm{E}$ et les croisements $\mathrm{B}$ et $\mathrm{C}$ a été significative sous le régime hydropotassique $\mathrm{NK} 0$, le croisement $\mathrm{E}$ a produit $0,65 \mathrm{~g}$ et $1,04 \mathrm{~g}$ par plant de moins que les croisements $B$ et $C$. Les différences de matière sèche racinaire entre les trois croisements sous stress hydrique n'ont pas été significatives pour les deux régimes potassiques (Tableau 5).

\section{Ramification des racines}

Les ramifications ont été influencées de façon très significative $(p<1 \%)$ par l'interaction génotype $x$ eau (Tableau 4). Le croisement $E$ a émis de façon significative moins de ramifications par plant que les croisements $B$ ( $39 \%$ ) et $\mathrm{C}$ ( $33 \%$ ) sous le régime hydrique $\mathrm{N}$, tandis que les différences entre les trois croisements n'ont pas été significatives sous stress hydrique (Tableau 6). Par ailleurs, le croisement $\mathrm{E}$ a en moyenne, émis sous stress hydrique, plus de ramifications par plant (40\%) que sous régime hydrique $\mathrm{N}$, pendant que le nombre de ramifications par plant sous stress hydrique par rapport au régime hydrique $\mathrm{N} \mathrm{a}$ baissé de 19,58 \% chez le croisement B (Tableau 6).

\section{EFFET DES TRAITEMENTS HYDRO- POTASSIQUES SUR LALONGUEURTOTALE DES RACINES SELON LE DIAMETRE}

\section{Racines de diamètre inférieur ou égale à $0,5 \mathrm{~mm}$}

La proportion du système racinaire constituée de racines extrafines $(0<\phi \leq 0,5 \mathrm{~mm})$ a été de $55 \%$ chez le croisement B et de $53 \%$ chez les croisements $\mathrm{C}$ et $\mathrm{E}$. La longueur totale des racines extrafines a été surtout influencée par l'interaction génotype $x$ sol (Tableau 4). Dans les tubes sans apport de $\mathrm{KCl}$, les croisements $B$ et $E$ ont de façon significative produit moins de racines extrafines que le croisement $C$, tandis que la différence entre les moyennes des trois croisements n'a pas été significative dans les tubes avec apport de $\mathrm{KCl}$ (Tableau 7). Par rapport aux tubes avec apport de $\mathrm{KCl}$, la longueur totale des racines extrafines dans les tubes sans apport de $\mathrm{KCl}$ a augmenté de $3,5 \mathrm{~m}(41,7 \%)$ chez le croisement $\mathrm{C}$, alors qu'elle a baissé de 1,3 m (13\%) chez le croisement B (Tableau 7).

Tableau 6 : Variation du nombre total de ramifications racinaires (centaine/plant) chez le palmier à huile selon l'origine génétique sous deux régimes hydriques.

Variation of root forks (hundred/plant) of oil palm crosses according to genetic origin and under two soil moisture regimes.

\begin{tabular}{lccc}
\hline Génétique origine & $\begin{array}{c}\text { Régime normal sans } \\
\text { déficit hydrique }(\mathrm{N})\end{array}$ & $\begin{array}{c}\text { Régime avec déficit } \\
\text { hydrique }(\mathrm{S})\end{array}$ & Moyenne \\
\hline $\mathrm{B}$ & $100,6 \mathrm{a}$ & $80,9 \mathrm{a}$ & 90,8 \\
$\mathrm{C}$ & $96,3 \mathrm{a}$ & $93,3 \mathrm{a}$ & 94,8 \\
$\mathrm{E}$ & $72,3 \mathrm{~b}$ & $101,4 \mathrm{a}$ & 86,9 \\
\hline Moyenne & 89,7 & 91,9 & 90,8 \\
\hline
\end{tabular}

Les valeurs qui portent des lettres différentes dans la même colonne sont différentes suivant le test de Student-Newman-Keuls à $5 \%$. B : DA115D x LM2T, C : DA8D x (LM9T x LM13T), E : (LM404 x DA10D) x LM2T 
Tableau 7 : Longueur totale $(\mathrm{m})$ des racines $0<\phi \leq \quad 0,5 \mathrm{~mm}$ selon l'origine génétique de trois croisements de palmier à huile en fonction du traitement potassique.

Total length $(\mathrm{m})$ of roots $0<\phi \leq 0,5 \mathrm{~mm}$ of three oil palm crosses according to genetic origin and potassium treatments.

\begin{tabular}{lccc}
\hline Origine génétique & Avec apport de $\mathrm{KCl}(\mathrm{K})$ & Sans apport de $\mathrm{KCl}(\mathrm{K} 0)$ & Moyenne \\
\hline $\mathrm{B}$ & $10,1 \mathrm{a}$ & $8,8 \mathrm{~b}$ & 9,4 \\
$\mathrm{C}$ & $8,4 \mathrm{a}$ & $11,9 \mathrm{a}$ & 10,1 \\
$\mathrm{E}$ & $8,6 \mathrm{a}$ & $8,6 \mathrm{~b}$ & 8,6 \\
\hline Moyenne & 9,8 & 9,0 & 9,4 \\
\hline
\end{tabular}

Les valeurs qui portent des lettres différentes dans la même colonne sont différentes suivant le test de StudentNewman-Keuls à $5 \%$. B : DA115D x LM2T, C : DA8D x (LM9T x LM13T), E : (LM404 x DA10D) x LM2T. $\phi$ : diamètre racinaire

Racines de diametre $0,5 \mathrm{~mm}<\phi \leq 1 \mathrm{~mm}$, $1 \mathrm{~mm}<\phi \leq 3 \mathrm{~mm}$ et $\phi>3 \mathrm{~mm}$

Le double déficit hydropotassique (SK0) a réduit de 1,81 à $2,87 \mathrm{~m}$ la longueur totale des racines très fines $(0,5 \mathrm{~mm}<\phi \leq 1 \mathrm{~mm})$ et de 0,73 à $1,91 \mathrm{~m}$ la longueur totale des racines fines (1 $\mathrm{mm}<\phi \leq 3 \mathrm{~mm}$ ) chez le croisement B par rapport aux régimes hydropotassiques NK et NKO (Tableau 8). Pour les deux classes de racines, les différences entre le croisement $E$ et les croisements $B$ et $C$ ont varié en fonction du régime hydropotassique (Tableau 8). Les différences entre les croisements $E$ et $B$ ont été en particulier élevées sous le régime hydropotassique NKO, où $\mathrm{B}$ a produit $3,38 \mathrm{~m}$ de racines très fines et $2,31 \mathrm{~m}$ de racines fines plus que $\mathrm{E}$. La différence de longueur des racines moyennes $(\phi>3 \mathrm{~mm})$ entre le croisement $C$ et les croisements $B$ et $E$ n'a été significative que sous le régime hydropotassique sans déficit hydrique avec apport du KCl (NK) (Tableau 8). Toutefois, la différence de longueur racinaire totale entre le croisement $C$ et les croisements $B$ et $E$ n'a été que de $0,21 \mathrm{~m}$ et de $0,19 \mathrm{~m}$.

Tableau 8 : Variation de la longueur $(\mathrm{m})$ des racines chez le palmier à huile sous 4 régimes hydropotassiques et selon l'origine génétique et le diamètre racinaire.

Variation of total roots length $(m)$ of oil palm under 4 hydropotassic regimes according to genetic origin and root diameter.

\begin{tabular}{|c|c|c|c|c|c|c|}
\hline \multirow{2}{*}{$\begin{array}{l}\text { Diamètre } \\
\text { racinaire }(\mathrm{mm})\end{array}$} & \multirow{2}{*}{$\begin{array}{l}\text { Origine } \\
\text { génétique }\end{array}$} & \multicolumn{2}{|c|}{$\begin{array}{c}\text { Régime normal sans déficit } \\
\text { hydrique }(\mathrm{N})\end{array}$} & \multicolumn{2}{|c|}{$\begin{array}{l}\text { Régime avec déficit } \\
\text { hydrique (S) }\end{array}$} & \multirow[t]{2}{*}{ Moyenne } \\
\hline & & Avec $\mathrm{KCl}(\mathrm{K})$ & Sans $\mathrm{KCl}(\mathrm{K} 0)$ & Avec KCl (K) & Sans $\mathrm{KCl}(\mathrm{K} 0)$ & \\
\hline \multirow{3}{*}{$0,5<\phi \leq 1$} & B & $6,06 a$ & $7,12 \mathrm{a}$ & $6,59 \mathrm{a}$ & $4,25 \mathrm{a}$ & 6,00 \\
\hline & $\mathrm{C}$ & $5,14 a b$ & $5,25 \mathrm{ab}$ & $4,64 a$ & $6,53 \mathrm{a}$ & 5,39 \\
\hline & $\mathrm{E}$ & $3,51 \mathrm{~b}$ & $3,74 b$ & $4,77 \mathrm{a}$ & $5,19 \mathrm{a}$ & 4,30 \\
\hline \multirow{3}{*}{$1<\phi \leq 3$} & B & $3,03 \mathrm{a}$ & $4,21 \mathrm{a}$ & $3,76 a$ & $2,30 \mathrm{a}$ & 3,32 \\
\hline & $\mathrm{C}$ & $3,24 a$ & $3,72 \mathrm{a}$ & $2,71 \mathrm{a}$ & $3,64 a$ & 3,33 \\
\hline & $\mathrm{E}$ & $2,62 \mathrm{a}$ & $1,90 \mathrm{~b}$ & $2,88 \mathrm{a}$ & $2,88 \mathrm{a}$ & 2,57 \\
\hline \multirow{3}{*}{$\phi>3$} & B & $0,05 \mathrm{~b}$ & $0,11 \mathrm{a}$ & $0,19 \mathrm{a}$ & $0,03 \mathrm{a}$ & 0,10 \\
\hline & $\mathrm{C}$ & $0,24 \mathrm{a}$ & $0,06 \mathrm{a}$ & $0,08 \mathrm{a}$ & $0,11 \mathrm{a}$ & 0,12 \\
\hline & E & $0,03 b$ & $0,03 a$ & $0,04 \mathrm{a}$ & $0,08 \mathrm{a}$ & 0,05 \\
\hline
\end{tabular}

Pour chaque classe de diamètre les valeurs qui portent des lettres différentes dans la même colonne sont différentes suivant le test de Student-Newman-Keuls à $5 \%$. B : DA115D x LM2T, C : DA8D x (LM9T x LM13T), E : (LM404 x DA10D) x LM2T. $\phi:$ diamètre racinaire 


\section{EFFET DES TRAITEMENTS POTASSIQUES SUR LE PROFIL RACINAIRE DES PLANTS ISSUS DES CROISEMENTS DE PALMIERA HUILE}

La figure 1 présente la surface totale de racines (SRT) des croisements en fonction de la profondeur et la dose de $\mathrm{KCl}$ (tout régime hydrique confondu). Cinquante quatre à $67 \%$ des racines ont été concentrés entre 0 et 30 $\mathrm{cm}$ en fonction des croisements et de la dose de $\mathrm{KCl}$. L'effet de l'application de $\mathrm{KCl}$ a été remarquable surtout au niveau du croisement B. Pour ce croisement, la concentration racinaire en surface $(0-30 \mathrm{~cm})$ a été de $54 \%$ sans apport de $\mathrm{KCl}$ et de $64 \%$ avec apport de $\mathrm{KCl}$. Cependant, sur l'ensemble du profil du sol, la surface racinaire par couche du sol diffère d'un croisement à un autre selon la profondeur du sol et la dose de $\mathrm{KCl}$. Dans les tubes sans apport potassique, la SRT du croisement $C$ a dépassé de $57,8 \mathrm{~cm}^{2}$ et de $80 \mathrm{~cm}^{2}$ celle des croisements $B$ et $E$ dans les horizons de 0 à $45 \mathrm{~cm}$. La différence entre les croisements $C$ et $B$ a été significative $(p<05 \%)$ dans la couche $15-30 \mathrm{~cm}$. Par contre, dans les tubes qui ont reçu le traitement $\mathrm{K}$, c'est le croisement $\mathrm{B}$ qui a poussé plus de racines sur tout le profil du sol jusqu'à $60 \mathrm{~cm}$ de profondeur. La surface totale de racines développées par le croisement $B$ entre 0 et $60 \mathrm{~cm}$ de profondeur a été de $411 \mathrm{~cm}^{2}$, pendant que celles des croisements $C$ et $E$ ont été respectivement de $327,9 \mathrm{~cm}^{2}$ et $301,9 \mathrm{~cm}^{2}$. La différence entre les trois croisements a été significative notamment, entre 0 et $30 \mathrm{~cm}$ en surface.

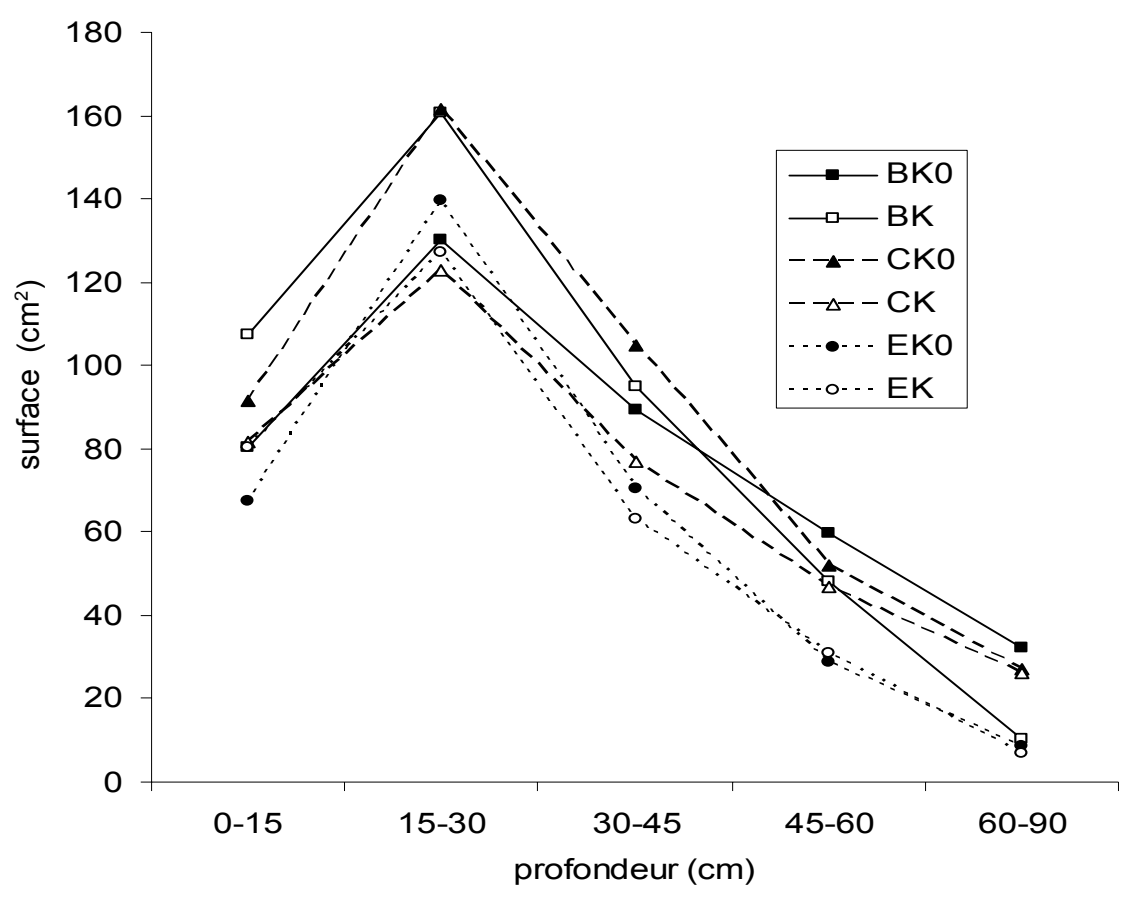

Figure 1 : Surface racinaire à différente profondeur du sol chez trois croisements de palmier à huile sous deux traitements potassiques.

Root surface area at different soil depths for thee oil palm crosses under two potassic treatments.

PPDS : $25,24 \mathrm{~cm}^{2} ; \mathrm{K} 0$ : sans apport de potassium, $\mathrm{K}$ : avec apport de potassium ; B : DA115D x LM2T, C : DA8D x (LM9T $x$ LM13T), E : (LM404 x DA10D) x LM2T 


\section{DISCUSSION}

\section{CLASSIFICATION DES RACINES SUIVANT LEUR GROSSEUR}

Les résultats de cette étude montrent que le système racinaire du palmier à huile au stade juvénile est composé en fonction du diamètre des racines de quatre classes. La première est constituée de racines extrafines (RXF) dont le diamètre ne dépasse pas $0,5 \mathrm{~mm}(0 \mathrm{~mm}<$ $\phi \leq 0,5 \mathrm{~mm}$ ). La deuxième classe est formée de racines très fines (RTF) de diamètre compris entre $0,5 \mathrm{~mm}$ et $1 \mathrm{~mm}(0,5 \mathrm{~mm}<\phi$ $\leq 1 \mathrm{~mm}$ ). La troisième classe regroupe des racines fines (RF) de diamètre variant entre 1 $\mathrm{mm}$ et $3 \mathrm{~mm}(1 \mathrm{~mm}<\phi \leq 3 \mathrm{~mm})$. La quatrième est composée de racines moyennes (RM) de diamètre supérieur à $3 \mathrm{~mm}(\phi>3 \mathrm{~mm})$. Les RXF et RTF sont assimilables aux racines latérales courtes $(0,2 \mathrm{~mm}<\phi<0,5 \mathrm{~mm})$, moyennes $(0,3 \mathrm{~mm}<\phi<0,6 \mathrm{~mm})$ et longues $(0,5 \mathrm{~mm}<\phi<1 \mathrm{~mm})$ observées par Jourdan (1995) et Jourdan et Rey (1997a). Tandis que les RF et RM sont assimilables aux racines primaires telles que la radicule $(1 \mathrm{~mm}<\phi<$ $2 \mathrm{~mm})$, les RI1-5 (2 $\mathrm{mm}<\phi<3 \mathrm{~mm})$ et les racines RI5-12 ( $3 \mathrm{~mm}<\phi<4 \mathrm{~mm}$ ) observées par ces mêmes auteurs. Toutefois, il est probable que les RF de diamètre compris entre $1 \mathrm{~mm}$ et $2 \mathrm{~mm}(1 \mathrm{~mm}<\phi<2 \mathrm{~mm})$ ne soient pas des radicules, puisque la durée maximale de vie des radicules du palmier est de 120 jours après germination (Jourdan, 1995). Il est possible que les conditions de culture aient influencé la morphologie des racines RI1-5 ou des «racines latérales longues». La modification du diamètre des racines selon les conditions de cultures, a été observée par différents auteurs (Anghinoni et Barber, 1980 ; Annerose, 1990). Les racines fines de diamètre $1 \mathrm{~mm}<\phi<2 \mathrm{~mm}$ peuvent être constituées de très fines racines RI1-5 ou/ et de grosses «racines latérales longues».

La catégorisation du système racinaire selon le diamètre est une pratique courante (Boukcim et al., 2001 ; Thongo M. Bou, 2003). Par exemple, Ran et al. (1994) ont identifié chez le pêcher trois classes de racines selon le diamètre. II s'agit des racines de diamètre inférieur à $1 \mathrm{~mm}(\phi<1 \mathrm{~mm})$, de diamètre compris entre $1 \mathrm{~mm}$ et $3 \mathrm{~mm}(1 \mathrm{~mm} \leq \phi \leq 3 \mathrm{~mm})$ et des racines dont le diamètre est supérieur à $3 \mathrm{~mm}(\phi>3 \mathrm{~mm})$. Ils considèrent comme racines fines, les racines au diamètre inférieur à $1 \mathrm{~mm}$. Chez le palmier au stade juvénile, il est plus approprié d'analyser en détail les racines de diamètre inférieur à $1 \mathrm{~mm}$, qui sont composées de plusieurs types de racines latérales (Jourdan et Rey, 1997a). De plus, les résultats ont mis en évidence des racines dont les diamètres sont inférieurs à $0,2 \mathrm{~mm}$ comme chez le maïs (Costa et al., 2000). Ces résultats montrent qu'il est possible de trouver chez le palmier à huile au stade juvénile des racines dont le diamètre est encore plus petit que les diamètres observés par Jourdan et al.(1997a). Toutefois, les racines de diamètre inférieur à $0,2 \mathrm{~mm}$ observées dans l'étude actuelle sont probablement des racines «latérales courtes». Jourdan (1995) trouve une relation positive entre le diamètre des racines du palmier et leur longueur.

\section{VARIABILITE DU DEVELOPPEMENT RACINAIRE}

Les différences de production de matière sèche totale ou de surface totale ou de longueur totale des racines entre croisements, semblent être liées principalement à des différences de racines très fines (RTF) et fines (RF). La longueur totale des RTF des croisements $B$ et $C$, représente $140 \%$ et $125 \%$ de celle du génotype E. Quant à la longueur totale des RF, elle représente $129 \%$ (B) et $130 \%$ (C). Pour les racines extrafines (RXF), les différences entre croisements ont été plus influencées par le niveau du traitement potassique. Les variations des divers axes racinaires ont comme effet un plus grand développement du système racinaire des croisements résistant $(C)$ et intermédiaire (B) que celui du croisement sensible (E). Ce résultat confirme l'existence d'une influence génétique sur le développement racinaire du palmier à huile au sein des croisements inter origines (Reis, 1991 ; Anonyme, 1992). Ce résultat est important, puisque l'aptitude du résistant $(\mathrm{C})$ à produire plus de racines que le sensible $(E)$, n'est pas antagoniste à la production de matière sèche de feuilles. La production de feuilles du croisement $C$, a été supérieure à celle du croisement $\mathrm{E}$ surtout au niveau des plants arrosés. 


\section{DIVERSITE ET PLASTICITE RACINAIRES SOUS DEFICIT HYDROPOTASSIQUE}

Des effets d'interactions significatives sont révélés entre l'origine génétique, la teneur du sol en potassium et l'humidité du sol sur la longueur totale et la surface totale des racines. Ces résultats montrent un comportement différentiel du système racinaire des trois croisements du palmier à huile en fonction de l'état hydropotassique du sol. Le croisement $C$ a augmenté la longueur et la surface totales des racines sur le sol moins riche en $\mathrm{K}$. Cet accroissement de surface et longueur racinaires s'est produit parallèlement à une augmentation significative des racines RXF. Ce résultat est comparable à l'augmentation de racines tertiaires et quaternaires observées sur des sols peu riches en K au champ (Ruer, 1968). La stimulation du développement racinaire face à la déficience minérale a été également rapportée chez d'autres espèces (Mackay et Barber, 1984 ; Mollier, 1999). Cette modification racinaire est souvent interprétée comme une adaptation des racines ou une manifestation de plasticité racinaire pour optimiser la capacité d'absorption minérale (Mollier, 1999). Cependant, Pellerin et al. (2000) ont montré qu'une carence en phosphate entraîne une réduction de la croissance racinaire chez le maïs au champ. Mollier (1999) précise qu'un déficit en phosphate stimule la croissance racinaire à court terme, mais la réduit à plus long terme. Les résultats suggèrent que la plasticité du système racinaire varie selon les croisements ou variétés mais dépend des conditions environnementales. L'effet stimulateur du déficit potassique sur le développement racinaire des croisements $B$ et $E$ semble dépendre de l'humidité du sol. Le déficit potassique a entraîné une réduction de surface racinaire du croisement $\mathrm{E}$ de $8 \%$ en régime sans déficit hydrique et une augmentation de $10 \%$ en régime hydrique limitant. En revanche, le déficit hydrique a induit une augmentation de la surface racinaire de $36 \%$ sous déficit potassique et de $13 \%$ sur un sol sans déficit potassique. L'augmentation de la MSR du croisement $E$ sous déficit hydrique indépendamment du traitement potassique est comparable aux résultats de Egilla et al. (2001). Ces auteurs ont observé une plus grande accumulation de matière sèche racinaire chez des plantules de Hibiscus rosa-sinensis L. cv., soumises à un déficit hydrique, qui n'est pas modifiée par un traitement au $\mathrm{K}_{2} \mathrm{SO}_{4}$. Le système racinaire du croisement $E$ a réagi donc plus au déficit hydrique qu'au déficit potassique. Le déficit hydrique a par contre réduit l'accumulation de matière sèche racinaire chez le croisement $C$ indépendamment de la fertilité du sol en K, tandis que l'effet du déficit hydrique sur la production de matière sèche racinaire chez $B$ dépend de la fertilité du sol en $K$. Ces résultats sont comparables aux observations de Khalfaoui (1994) chez le mil et le sorgho et de Annerose (1990) chez l'arachide. Ces auteurs ont observé des effets d'interactions génotypes/régimes hydriques significatives sur le système racinaire de plusieurs variétés chez le mil, le sorgho et l'arachide. Ces variations de comportements pourraient s'expliquer par une intensification de développement de certains types de racines.

Un double déficit hydropotassique a entrainé une augmentation de la surface racinaire du croisement $E$ grâce à une plus grande croissance des RXF. Inversement, il a réduit la surface racinaire du croisement $B$ par diminution de la longueur totale des RTF et RF. Lorsque l'eau n'est pas un facteur limitant, le système racinaire du croisement $B(+28 \%$ SRT) s'est montré plus plastique que celui du croisement C (+10,5\% SRT) au déficit potassique. Cet accroissement de surface racinaire de $B$, semble être liée à une plus grande croissance des RTF $(118 \%)$ et des RF (139\%), par rapport à leur longueur sous le régime hydrique sans déficit, avec apport de K. Par contre, la plasticité racinaire de $C$ face au déficit potassique sous régime hydrique $\mathrm{N}$, semble être liée à un plus grand accroissement des RXF et RF dont les longueurs représentent $136 \%$ et $115 \%$ de leur longueur respective sous condition optimale. Les deux croisements $B$ et $C$, en augmentant leur surface racinaire face au déficit de $\mathrm{K}$, ont modifié différemment leur architecture racinaire, ceci pourrait influencer leur capacité d'extraction hydrique et d'absorption minérale. Par ailleurs, les résultats suggèrent qu'un régime de double déficit hydropotassique en pépinière serait particulièrement dommageable pour la croissance racinaire du croisement $B$ et probablement pour la croissance générale des plants.

\section{CONCLUSION}

La technique d'analyse d'image racinaire par WinRhizo, a permis de montrer que des différences significatives caractérisent le 
système racinaire plants issus des 3 croisements de palmier à huile au stade juvénile. Le croisement sensible à la sécheresse a un système racinaire moins développé que celui des croisements intermédiaire et résistant. Toutefois, les résultats montrent que le système racinaire du palmier à huile est sensible aux effets d'interactions entre croisements et les disponibilités en potassium et en eau du sol. La manifestation de ce phénomène a modifié le classement des croisements pour le développement du système racinaire en fonction du régime hydropotassique. Le système racinaire du croisement $B$ a été particulièrement réduit par le double déficit hydropotassique.

\section{REFERENCES}

Anghinoni I. and S. A. Barber. 1980. Phosphorus influx and growth characteristics of corn roots as influenced by phosphorus supply. Agronomy Journal $72: 685$ - 688 .

Annerose D. J. 1990 Recherches sur les mécanismes physiologiques d'adaptation à la sécheresse. Application au cas de l'arachide (Arachis hypogaea L) cultivée au Sénégal. Thèse de Doctorat, Université Paris VII (France), 282 p.

Anonyme. 1992. Palmier à huile. IRHO - CIRAD. Oléagineux 47 (6) : 289 - 328.

Boukcim H., L. Pages, C. Plassard and D. Mousain. 2001. Root system architecture and receptivity to mycorrhizal infection in seedlings of Cedrus atlantica as affected by nitrogen source and concentration. Tree Physiology $21: 109$ - 115.

Chopart J. L. and P. Siband. 1999. Development and validation of a model to describe root length density of maize from root counts on soil profiles. Plant and soil $214: 61$ - 74.

Cornaire B., C. Daniel, Y. Zuily-Fodil and E. Lamade. 1994. Le comportement du palmier à huile sous stress hydrique. Données du problème, premiers résultats et voies de recherche. Oléagineux $49: 1$ 12.

Corrado F. et W. Wuidart. 1990. Germination des graines de palmier à huile (Elaeis guineensis Jacq.) en sacs de polyéthylène - Méthode par "chaleur sèche". Oléagineux 45 (11) : 511 - 514.

Costa C., L. M. Dwyer, R. I. Hamilton, C. Hamel, L. Nantais and D. L. Smith. 2000. A sampling method for measurement of large root systems with scanner-based image analysis. Agronomy Journal 92 (4): $621-627$.

Danjon F., D. Pot and F. Courdier. 2000. Genetics of root architecture in 1- year - old Pinus pinaster measured with the WinRHIZO image analysis system : prelimary results. In : A. Stokes (Ed.). The supporting roots of trees and woody plants: Form, Function and Physiology. Kluwer Academic Publishers. Netherlands: pp 77 - 81.

Davies W. J. and M. A. Bacon. 2003. Adaptation of roots to drought. In : de Kroon H. and E. J. W. Visser (Eds.). Root ecology. Ecological studies 168, Springer Verlag, Berlin, Heidelberg : pp. 173 - 192.

Egilla J. N., F. T. Davies and Jr. M. C. Drew. 2001. Effect of potassium on drought resistance of Hibiscus rosa-sinensis cv. Leprechaun : Plant growth, leaf macro- and micronutrient content and root longevity. Plant and Soil $229: 213-224$.

Huang B. and H. Gao. 2000. Root physiological characteristics associated with drought resistance in tall fescue cultivars. Crop Science $40: 196$ - 203

Jourdan C. 1995. Modélisation de l'architecture et du développement du système racinaire du palmier à huile (Elaeis guineensis Jacq.). Thèse de Doctorat, Université. Montpellier II (France), 243 p.

Jourdan C., H. Rey and Y. Guédon. 1995. Architectural analysis and modelling of branching process of the young oil palm root system. Plant and Soil $177: 63$ - 72.

Jourdan C. and H. Rey. 1997a. Architecture and development of the oil-palm (Elaeis guineensis Jacq.) root system. Plant and Soil $189: 33$ - 48 .

Jourdan C. and H. Rey. 1997b. Modelling and simulation of the architecture and development of the oil-palm (Elaeis guineensis Jacq.) root system - II. Estimation of root parameters using the Racines postprocessor. Plant and soil 190 : $235-246$

Khalkfaoui J.-L. B. 1994. Premiers acquis du CERAAS sur la génétique et la sélection de l'adaptation à la sécheresse des espèces cultivées. Cas de l'étude des paramètres du système racinaire. In : Reyniers $F$. N. and L. Netoyo (Eds.). Bilan hydrique Agricole et sécheresse en Afrique tropicale vers une gestion des flux hydriques par le système de culturex. Séminaire international 
Bamako 9 - 13 décembre 1991. John Libbey Eurotext., Paris : pp $151-159$

Khalid H., Z. Z. Zin and J. M. Anderson. 1999. Quantification of oil palm biomass and nutrient value in a mature plantation. II. Below-ground biomass. Journal of Oil Palm Research $11(2): 63-71$

Kolek J. and V. Kozinka. 1992. Physiology of the plant root system 46. Kluwer Academic publishers, Dordrecht, Netherlands 361 p.

Mackey A. D. and S. A. Barber. 1984. Comparison of root and root hair growth in solution and soil culture. Journal of plant nutrition 7 (12) : 1745 - 1757.

Mollier A. 1999. Croissance racinaire du maïs (Zea mays L.) sous déficience en phosphore Etude expérimentale et modélisation. Thèse de Doctorat, Université Paris XI Orsay, France, 192 p.

Nodichao L. 1997. Biodiversité racinaire et résistance à la sécheresse. M. Sc, Université Laval Canada, 168 p.

Pardo A. and M. Amato. 2000. Relationships between soil structure root distribution and water uptake of chickpea (Cicer arietinum L.) - Plant growth and water distribution. European Journal of Agronomy $13: 39$ - 45.

Pellerin S., A. Mollier and D. Plénet. 2000. Phosphorus deficiency affects the rate of emergence and number of maize adventitious nodal roots. Agronomy Journal $92: 690-697$

Quencez P. and G. de Taffin. 1981. Relation entre la nutrition potassique et la pluviométrie en culture de palmiers à huile et cocotiers. Oléagineux 36 (1) : 1 - 5 .
Ran Y., R. Habib, B. Bas-Yosef and A. Erez. 1994. Root volume effects on nitrogen uptake and partitioning in peach trees. Agronomy Journal 86 (3) : 530 - 534.

Régent Instruments Inc. 2001. Mac/WinRhizoTM, 5.0a Reference, $59 \mathrm{p}$.

Reis de Carvalho C. J. 1991. Mécanismes de résistance à la sécheresse chez des plantes jeunes et adultes de palmier à huile (Elaeis guineensis Jacq.). Thèse de Doctorat, Université de Paris - Orsay (France), $203 \mathrm{p}$.

Ruer P. 1968. Contribution à l'étude du système racinaire du palmier à huile. Thèse de Doctorat Université de Paris, France, $117 \mathrm{p}$.

Sinclair T. R. and M. M. Ludlow. 1986. Influence of soil water supply on the plant water balance of four tropical grain legumes. Australia J. plant Physiology $13: 329$ - 341 .

Tailliez B. 1971. Le système racinaire du palmier à huile sur la plantation de San Alberto Colombie. Oléagineux 26 : 435 - 447.

Thongo-M'Bou A. 2003. Caractérisation de la dynamique de croissance racinaire dans une chronoséquence d'Eucalyptus au Congo. Mémoire de DEA. Faculté des sciences Marien Ngouabi Brazzaville (Congo), $43 \mathrm{p}$.

Tinker P. B. and P. H. Nye. 2000. Solute movement in the rhizosphere. New York Oxford, Oxford University Press. 444 p.

Vandeleur R., C. Niemietz, J. Tilbrook and S. D. Tyerman. 2005. Roles of aquaporins in root responses to irrigation. Plant and Soil 274 : $141-161$. 